\title{
Matter density perturbations and effective gravitational constant in modified gravity models of dark energy
}

\author{
Shinji Tsujikawa ${ }^{1, *}$ \\ ${ }^{1}$ Department of Physics, Gunma National College of Technology, Gunma 371-8530, Japan
}

(Dated: February 11, 2013)

\begin{abstract}
We derive the equation of matter density perturbations on sub-horizon scales for a general Lagrangian density $f(R, \phi, X)$ that is a function of a Ricci scalar $R$, a scalar field $\phi$ and a kinetic term $X=-(\nabla \phi)^{2} / 2$. This is useful to constrain modified gravity dark energy models from observations of large-scale structure and weak lensing. We obtain the solutions for the matter perturbation $\delta_{m}$ as well as the gravitational potential $\Phi$ for some analytically solvable models. In a $f(R)$ dark energy model with the Lagrangian density $f(R)=\alpha R^{1+m}-\Lambda$, the growth rates of perturbations exhibit notable differences from those in the standard Einstein gravity unless $m$ is very close to 0 . In scalartensor models with the Lagrangian density $f=F(\phi) R+2 p(\phi, X)$ we relate the models with coupled dark energy scenarios in the Einstein frame and reproduce the equations of perturbations known in the current literature by making a conformal transformation. We also estimate the evolution of perturbations in both Jordan and Einstein frames when the energy fraction of dark energy is constant during the matter-dominated epoch.
\end{abstract}

PACS numbers: 98.80.-k

\section{INTRODUCTION}

Recent observations have determined basic cosmological parameters in high-precisions, but at the same time they posed a serious problem about the origin of dark energy (DE). The analysis of Super-Nova Ia (SNIa) [1] is based upon the background expansion history of the universe around the redshift $z<\mathcal{O}(1)$. The constraint obtained from SNIa so far has a degeneracy in the equation of state (EOS) of DE [2]. To many people's frustration, the $\Lambda \mathrm{CDM}$ model with an $\mathrm{EOS} w_{\mathrm{DE}}=-1$ has been continuously favored from observations. This degeneracy has been present even adding other constraints coming from Cosmic Microwave Background (CMB) [3] and Baryon Acoustic Oscillations (BAO) [4].

The models of dark energy can be broadly classified into two classes $[5,6]$. The first corresponds to introducing a specific matter that leads to an accelerated expansion. Most of scalar field models such as quintessence [7] and k-essence [8] belong to this class. The second class corresponds to so-called modified gravity models such as $f(R)$ gravity [9], scalar-tensor theories [10] and braneworld models [11]. In order to break the degeneracy of observational constraints on $w_{\mathrm{DE}}$ and to discriminate between a host of DE models, it is important to find additional information other than the background expansion history of the Universe. In this paper we will show that modified gravity models can be distinguished from others by considering the evolution of matter perturbations $\delta_{m}$ and gravitational potentials $\Phi$.

In Einstein gravity it is well known that linear matter perturbations on sub-horizon scales satisfy the following

*Electronic address: shinji@nat.gunma-ct.ac.jp equation

$$
\ddot{\delta}_{m}+2 H \dot{\delta}_{m}-4 \pi G \rho_{m} \delta_{m}=0,
$$

where $H$ is a Hubble parameter, $G$ is a Newton's gravitational constant, $\rho_{m}$ is an energy density of the nonrelativistic matter, and a dot represents a derivative with respect to cosmic time $t$. During the matter-dominated epoch this has a growing-mode solution $\delta_{m} \propto a \propto t^{2 / 3}$, which leads to the formation of large-scale structure. In modified gravity models the growth rates of perturbations are different because of the modification of the gravitational constant as well as the change of the background evolution. In the context of $f(R)$ gravity, in particular, there have been a number of recent works about the evolution of density perturbations during the matterdominated and dark energy dominated epochs [12].

We will derive the equation of matter perturbations in Sec. III for a very general Lagrangian density $f(R, \phi, X)$, where $R$ is a Ricci scalar and $\phi$ is a scalar field with a kinetic term $X=-(\nabla \phi)^{2} / 2$. Together with a sub-horizon approximation we assume that $F \equiv \partial f / \partial R$ depends on $\phi$ and $R$ but not on $X$. In fact this Lagrangian covers most of modified gravity DE scenarios such as $f(R)$ gravity models and scalar-tensor theories. The effect of modified gravity appears in an effective gravitational constant $G_{\text {eff }}$ whose explicit form is given in Eq. (40). We derive a parameter $\eta$ introduced in Ref. [13] to quantify the strength of an anisotropic stress and also evaluate a parameter $\Sigma=q(1+\eta / 2)$, where $q$ is a quantity that characterizes the deviation from the gravitational constant measured in solar system experiments today.

The results in this paper can be important for future surveys of weak lensing [14] as well as for the observations of large-scale structure (LSS) [15]. In Ref. [16] the deviation from Einstein gravity was constrained from the galaxy clustering by taking into account an additional Yukawa correction to the gravitational constant. It will 
be possible to carry out similar observational constraints on our $f(R, \phi, X)$ DE models from the LSS data by solving the equation of matter perturbations. In Ref. [13] the authors proposed a DE parametrization using the variables $(\Sigma, \eta)$ together with a linear perturbation growth factor $\gamma$ introduced in Refs. [17-19]. If the deviation from the Einstein gravity case $(\Sigma, \eta)=(1,0)$ is detected from future survey of weak lensing, this allows us to distinguish modified gravity models from the models in Einstein gravity.

In Sec. IV we will find solutions for $\delta_{m}$ and $\Phi$ during the matter-dominated epoch for some analytically solvable models. In particular we show that $f(R)$ dark energy models have a peculiar scale-dependence of perturbations unlike the case of Einstein gravity. The effect of modified gravity on perturbations is important provided that a dimensionless variable $m=R f_{, R R} / f_{, R}$, which characterizes the deviation from the $\Lambda$ CDM model, is not very close to 0 .

The scalar-tensor models with the Lagrangian density $f=F(\phi) R+2 p(\phi, X)$ correspond to coupled dark energy models in the Einstein frame with a coupling $Q(\phi)=-F_{, \phi} / 2 F$. In Sec. $\mathrm{V}$ we derive the equation of matter perturbations in the Einstein frame under a conformal transformation and show that this in fact coincides with the equation in the models of dark energy coupled to the matter [20]. We also derive the growth rates of perturbations in both Jordan and Einstein frames for the models in which the so-called $\phi$-matter dominated epoch [21] is present.

\section{BACKGROUND EQUATIONS}

We start with the following 4-dimensional action

$$
S=\int \mathrm{d}^{4} x \sqrt{-g}\left[\frac{1}{2} f(R, \phi, X)+\mathcal{L}_{m}\right],
$$

where $g$ is a determinant of a metric $g_{\mu \nu}, f$ is a function in terms of a Ricci scalar $R$, a scalar field $\phi$ and a kinetic term $X=-\phi^{, c} \phi_{, c} / 2$. $\mathcal{L}_{m}$ is a Lagrangian density for a pressureless matter whose energy density is given by $\rho_{m}$. We use the metric signature $(-,+,+,+)$.

The gravitational field equation and the equation of motion of the field $\phi$ are given by

$$
\begin{aligned}
F G_{\mu \nu}= & \frac{1}{2}(f-R F) g_{\mu \nu}+F_{, \mu ; \nu}-\square F g_{\mu \nu} \\
& +\frac{1}{2} f_{, X} \phi_{, \mu} \phi_{, \nu}+T_{\mu \nu}^{(m)}, \\
\left(f_{, X} \phi^{, c}\right)_{; c}+f_{, \phi}=0 &
\end{aligned}
$$

where $F=\partial f / \partial R, G_{\mu \nu}$ is an Einstein tensor, and $T_{\mu \nu}^{(m)}$ is an energy-momentum tensor of the pressureless matter.

In a flat Friedmann-Robertson-Walker (FRW) metric with a scale factor $a$, we obtain the following background equations

$$
\begin{aligned}
& 3 F H^{2}=f_{, X} X+\frac{1}{2}(F R-f)-3 H \dot{F}+\rho_{m} \\
& -2 F \dot{H}=f_{, X} X+\ddot{F}-H \dot{F}+\rho_{m} \\
& \frac{1}{a^{3}}\left(a^{3} \dot{\phi} f_{, X}\right)-f_{, \phi}=0 \\
& \dot{\rho}_{m}+3 H \rho_{m}=0
\end{aligned}
$$

where $H \equiv \dot{a} / a, R=6\left(2 H^{2}+\dot{H}\right)$, and a dot represents a derivative with respect to cosmic time $t$.

In order to confront the DE equation of state with observations such as SNIa, we rewrite Eqs. (5) and (6) as follows:

$$
\begin{aligned}
& 3 F_{0} H^{2}=\rho_{\mathrm{DE}}+\rho_{m}, \\
& -2 F_{0} \dot{H}=\rho_{\mathrm{DE}}+p_{\mathrm{DE}}+\rho_{m},
\end{aligned}
$$

where

$$
\begin{aligned}
\rho_{\mathrm{DE}} & =\frac{1}{2}(F R-f)-3 H \dot{F}+f_{, X} X+3 H^{2}\left(F_{0}-F\right),(11) \\
p_{\mathrm{DE}} & =\ddot{F}+2 H \dot{F}-\frac{1}{2}(F R-f)-\left(2 \dot{H}+3 H^{2}\right)\left(F_{0}-F\right) .
\end{aligned}
$$

Here the subscript "0" represents present values. It is easy to show that $\rho_{\mathrm{DE}}$ and $p_{\mathrm{DE}}$ defined in this way satisfy the usual energy conservation equation

$$
\dot{\rho}_{\mathrm{DE}}+3 H\left(\rho_{\mathrm{DE}}+p_{\mathrm{DE}}\right)=0,
$$

where we used Eq. (7). This was already shown to hold in the context of the scalar-tensor gravity [22, 23] as well as the $f(R)$ gravity [24]. We define the DE equation of state as

$$
\begin{aligned}
w_{\mathrm{DE}} & \equiv \frac{p_{\mathrm{DE}}}{\rho_{\mathrm{DE}}} \\
& =-1+\frac{2 f_{, X} X+2 \ddot{F}-4 H \dot{F}-4 \dot{H}\left(F_{0}-F\right)}{2 f_{, X} X+F R-f-6 H \dot{F}+6 H^{2}\left(F_{0}-F\right)} .
\end{aligned}
$$

Integrating Eq. (8) gives

$$
\rho_{m}=3 F_{0} \Omega_{m}^{(0)} H_{0}^{2}(1+z)^{3},
$$

where $z=a_{0} / a-1$ is a redshift and $\Omega_{m}^{(0)}$ is a present energy fraction of the non-relativistic matter. Then by using Eqs. (9) and (10) the equation of state $w_{\mathrm{DE}}$ can be expressed as

$$
w_{\mathrm{DE}}=-\frac{3 r-(1+z)(\mathrm{d} r / \mathrm{d} z)}{3 r-3 \Omega_{m}^{(0)}(1+z)^{3}},
$$

where $r=H^{2}(z) / H_{0}^{2}$. This is the same relation as the one derived in Einstein gravity [6]. Thus $w_{\mathrm{DE}}$ is constrained in the usual way from SNIa observations. From Eq. (14) we find that the evolution of $w_{\mathrm{DE}}$ depends upon 
the models of dark energy. Hence one can test the viability of the models by confronting $w_{\mathrm{DE}}$ with observations.

If the scalar field $\phi$ is minimally coupled gravity, e.g., $f=R / 8 \pi G+2 p(\phi, X)$, the structure of the Lagrangian density $p(\phi, X)$ can be reconstructed by the evolution of the Hubble parameter $H(z)$ [25]. For the models where the field $\phi$ is coupled to gravity or the models in which the Lagrangian includes non-linear terms in $R$, we need additional information to determine the strength of gravitational couplings. This can be provided by considering the evolution of matter density perturbations.

\section{PERTURBATION EQUATIONS}

We consider the following perturbed metric with scalar metric perturbations $\Phi$ and $\Psi$ in a longitudinal gauge:

$$
\mathrm{d} s^{2}=-(1+2 \Phi) \mathrm{d} t^{2}+a^{2}(1-2 \Psi) \delta_{i j} \mathrm{~d} x^{i} \mathrm{~d} x^{j} .
$$

We decompose the field into the background and inhomogeneous parts: $\phi=\tilde{\phi}(t)+\delta \phi(t, \mathbf{x})$. In what follows we drop the tilde for simplicity. The energy momentum tensors of the non-relativistic matter are decomposed as $T_{0}^{0}=-\left(\rho_{m}+\delta \rho_{m}\right)$ and $T_{\alpha}^{0}=-\rho_{m} v_{m, \alpha}$, where $v_{m}$ is a velocity potential.
The Fourier transformed perturbation equations are given by [26]

$$
\begin{aligned}
& 3 H(\dot{\Psi}+H \Phi)+\frac{k^{2}}{a^{2}} \Psi+\frac{1}{2 F}\left[-\frac{1}{2}\left(f_{, \phi} \delta \phi+f_{, X} \delta X\right)+\frac{1}{2} \dot{\phi}^{2}\left(f_{, X \phi} \delta \phi+f_{, X X} \delta X\right)+f_{, X} \dot{\phi} \delta \dot{\phi}-3 H \delta \dot{F}\right. \\
& \left.+\left(3 H^{2}+3 \dot{H}-\frac{k^{2}}{a^{2}}\right) \delta F+3 \dot{F}(\dot{\Psi}+H \Phi)+\left(3 H \dot{F}-f_{, X} \dot{\phi}^{2}\right) \Phi+\delta \rho_{m}\right]=0 \\
& f_{, X}\left[\delta \ddot{\phi}+\left(3 H+\frac{\dot{f}, X}{f_{, X}}\right) \delta \dot{\phi}+\frac{k^{2}}{a^{2}} \delta \phi-\dot{\phi}(3 \dot{\Psi}+\dot{\Phi})\right]-2 f_{, \phi} \Phi+\frac{1}{a^{3}}\left(a^{3} \dot{\phi} \delta f_{, X}\right) \cdot \delta f_{, \phi}=0 \\
& \Psi=\Phi+\frac{\delta F}{F} \\
& \delta \dot{\rho}_{m}+3 H \delta \rho_{m}=\rho_{m}\left(3 \dot{\Psi}-\frac{k^{2}}{a} v_{m}\right) \\
& \dot{v}_{m}+H v_{m}=\frac{1}{a} \Phi
\end{aligned}
$$

where $k$ is a comoving wavenumber.

We define the gauge-invariant matter density perturbation $\delta_{m}$, as

$$
\delta_{m} \equiv \frac{\delta \rho_{m}}{\rho_{m}}+3 H v, \quad \text { where } \quad v \equiv a v_{m} .
$$

Then Eqs. (21) and (22) yield

$$
\begin{aligned}
& \dot{\delta}_{m}=-\frac{k^{2}}{a^{2}} v+3(\Psi+H v) \\
& \dot{v}=\Phi
\end{aligned}
$$

from which we obtain

$$
\ddot{\delta}_{m}+2 H \dot{\delta}_{m}+\frac{k^{2}}{a^{2}} \Phi=3 \ddot{B}+6 H \dot{B},
$$

where $B \equiv \Psi+H v$.

Following the approach in Ref. [6, 22, 27], we use a subhorizon approximation under which the leading terms correspond to those containing $k^{2}$ and $\delta_{m}\left(\right.$ or $\left.\delta \rho_{m}\right)$ in Eq. (26) and also in Eqs. (18)-(19). Basically the terms on the r.h.s. of Eq. (26) give the contribution of the order $H^{2} \Psi$, which implies that they are negligible relative to the term $\left(k^{2} / a^{2}\right) \Phi$ for the modes deep inside the Hubble radius $\left(k^{2} \gg a^{2} H^{2}\right)$.

If the mass $m_{\phi}$ of the field perturbation $\delta \phi$ is larger than the term $k / a$ then we need to take into account this mass term. The expression of $m_{\phi}$ was derived in Ref. [20] in coupled dark energy models with the Lagrangian density $p(\phi, X)$. In Einstein gravity with a standard scalar field the mass squared is given by $m_{\phi}^{2}=V_{, \phi \phi}-2 \dot{\phi}^{2}$. When the field $\phi$ is responsible for dark energy the terms $V_{, \phi \phi}$ and $2 \dot{\phi}^{2}$ are of order $H^{2}$ or less, which then gives $\left|m_{\phi}\right| \lesssim H$. Hence the approximation neglecting the mass term $m_{\phi}$ relative to $k / a$ is justified in such a model. There may be some specific $f(R, \phi, X)$ models in which the condition $\left|m_{\phi}\right| \ll k / a$ is violated, but we do not con- 
sider such cases.

Then Eq. (26) is approximately given by

$$
\ddot{\delta}_{m}+2 H \dot{\delta}_{m}+\frac{k^{2}}{a^{2}} \Phi \simeq 0 .
$$

The next step is to express $\Phi$ in terms of $\delta_{m}$. From Eq. (18) we find

$$
\frac{k^{2}}{a^{2}} \Psi \simeq \frac{1}{2 F}\left(\frac{k^{2}}{a^{2}} \delta F-\delta \rho_{m}\right) .
$$

Eliminating the term $\Psi$ by using Eq. (20) gives

$$
\frac{k^{2}}{a^{2}} \Phi \simeq-\frac{k^{2}}{2 a^{2}} \frac{\delta F}{F}-\frac{1}{2 F} \delta \rho_{m} .
$$

In what follows we shall study the case in which $F$ depends on $\phi$ and $R$ but not on $X$, i.e.,

$$
F=F(\phi, R)
$$

This actually includes most of dark energy models proposed in the current literature. Then $\delta F$ in Eq. (29) is given by

$$
\delta F=F_{, \phi} \delta \phi+F_{, R} \delta R
$$

where $\delta R$ is

$$
\begin{aligned}
\delta R= & 2\left[-3\left(\ddot{\Psi}+4 H \dot{\Psi}+H \dot{\Phi}+\dot{H} \Phi+4 H^{2} \Phi\right)\right. \\
& \left.+\left(\frac{k^{2}}{a^{2}}-3 \dot{H}\right) \Phi-2 \frac{k^{2}}{a^{2}} \Psi\right] \\
\simeq & -2 \frac{k^{2}}{a^{2}}\left(\Phi+2 \frac{\delta F}{F}\right) .
\end{aligned}
$$

Again we used the fact that the first five terms in Eq. (32) are of order $H^{2} \Phi, H^{2} \Psi$ or less. Plugging Eq. (31) into Eq. (32), we find

$$
\delta R \simeq-\frac{2 k^{2}}{a^{2}} \frac{\Phi+\frac{2 F_{, \phi}}{F} \delta \phi}{1+4 \frac{k^{2}}{a^{2}} \frac{F, R}{F}} .
$$

Taking notice that $\delta R$ includes the term $k^{2} / a^{2}$, the quantity $\delta f$ is approximately given by

$$
\begin{aligned}
\delta f & =f_{, \phi} \delta \phi+f_{, X} \delta X+f_{, R} \delta R \\
& \simeq F \delta R .
\end{aligned}
$$

Then from Eq. (19) we find

$$
f_{, X} \frac{k^{2}}{a^{2}} \delta \phi-F_{, \phi} \delta R \simeq 0,
$$

which leads to the following relation

$$
\delta \phi \simeq-2 \frac{F_{, \phi}}{f_{, X}\left(1+4 \frac{k^{2}}{a^{2}} \frac{F, R}{F}\right)+\frac{4 F_{, \phi}^{2}}{F}} \Phi .
$$

Plugging this into Eq. (32), we get

$$
\delta R \simeq-\frac{2 k^{2}}{a^{2}} \frac{f_{, X}}{f_{, X}\left(1+4 \frac{k^{2}}{a^{2}} \frac{F, R}{F}\right)+\frac{4 F_{, \phi}^{2}}{F}} \Phi .
$$

From Eqs. (29), (31), (36) and (37) the gravitational potential $\Phi$ is expressed as

$$
\frac{k^{2}}{a^{2}} \Phi \simeq-\frac{\rho_{m}}{2 F} \frac{f_{, X}+4\left(f_{, X} \frac{k^{2}}{a^{2}} \frac{F, R}{F}+\frac{F_{, \phi}^{2}}{F}\right)}{f_{, X}+3\left(f_{, X} \frac{k^{2}}{a^{2}} \frac{F, R}{F}+\frac{F_{, \phi}^{2}}{F}\right)} \delta_{m},
$$

where we used $\delta_{m} \simeq \delta \rho_{m} / \rho_{m}$ under the sub-horizon approximation. Hence the equation (27) of matter perturbations yields

$$
\ddot{\delta}_{m}+2 H \dot{\delta}_{m}-4 \pi G_{\text {eff }} \rho_{m} \delta_{m} \simeq 0,
$$

where the effective gravitational "constant" on subhorizon scales is given by

$$
G_{\mathrm{eff}} \simeq \frac{1}{8 \pi F} \frac{f_{, X}+4\left(f_{, X} \frac{k^{2}}{a^{2}} \frac{F_{, R}}{F}+\frac{F_{, \phi}^{2}}{F}\right)}{f_{, X}+3\left(f_{, X} \frac{k^{2}}{a^{2}} \frac{F_{, R}}{F}+\frac{F_{, \phi}^{2}}{F}\right)} .
$$

From Eq. (38) the gravitational potential is

$$
\Phi \simeq-4 \pi G_{\mathrm{eff}} \frac{a^{2}}{k^{2}} \rho_{m} \delta_{m},
$$

which corresponds to a Poisson equation in the Fourier space. In what follows we use the standard equality rather than the approximate equality $(\simeq)$ for the results obtained under the sub-horizon approximation.

We also define a parameter $\eta$ that characterizes the strength of an anisotropic stress:

$$
\eta \equiv \frac{\Phi-\Psi}{\Psi} .
$$

Using Eqs. (20), (36) and (37) we obtain

$$
\eta=\frac{2 f_{, X} \frac{k^{2}}{a^{2}} \frac{F, R}{F}+\frac{2 F_{, \phi}^{2}}{F}}{f_{, X}\left(1+\frac{2 k^{2}}{a^{2}} \frac{F_{, R}}{F}\right)+\frac{2 F_{, \phi}^{2}}{F}} .
$$

The gravitational potential $\Psi$ satisfies

$$
\frac{k^{2}}{a^{2}} \Psi=-\frac{\rho_{m}}{2 F} \frac{f_{, X}+2\left(f_{, X} \frac{k^{2}}{a^{2}} \frac{F, R}{F}+\frac{F_{, \phi}^{2}}{F}\right)}{f_{, X}+3\left(f_{, X} \frac{k^{2}}{a^{2}} \frac{F, R}{F}+\frac{F_{, \phi}^{2}}{F}\right)} \delta_{m} .
$$

We define another parameter $q$ via $\left(k^{2} / a^{2}\right) \Psi=$ $-4 \pi G_{0} q \rho_{m} \delta_{m}$, where $G_{0}$ is a gravitational constant measured in the solar system experiments today. Then $q$ is given by

$$
q=\frac{1}{8 \pi F G_{0}} \frac{f_{, X}+2\left(f_{, X} \frac{k^{2}}{a^{2}} \frac{F_{, R}}{F}+\frac{F_{, \phi}^{2}}{F}\right)}{f_{, X}+3\left(f_{, X} \frac{k^{2}}{a^{2}} \frac{F_{, R}}{F}+\frac{F_{, \phi}^{2}}{F}\right)} .
$$


Defining a combination of parameters, $\Sigma=q(1+\eta / 2)$, we obtain

$$
\Sigma=\frac{1}{8 \pi F G_{0}} .
$$

This agrees with the result in Ref. [13] derived in the specific scalar-tensor model: $f=F(\phi) R+2 X-2 V(\phi)^{1}$. For this model the parameter $\eta$ reduces to $\eta=F_{, \phi}^{2} /(F+$ $\left.F_{, \phi}^{2}\right)$, which again agrees with the result given in Ref. [13].

In order to confront the modified gravity models with the observations of weak lensing, we use the fact that the potential that characterizes the deviation of light rays corresponds to $\Phi_{\mathrm{WL}} \equiv \Phi+\Psi$ [28]. From Eqs. (38), (44) and (46) we find that the lensing potential satisfies

$$
\Phi_{\mathrm{WL}} \simeq-8 \pi G_{0} \frac{a^{2}}{k^{2}} \rho_{m} \delta_{m} \Sigma .
$$

The effect of modified gravity theories manifests themselves in weak lensing in at least two ways. One is the multiplication of the term $\Sigma$ on the r.h.s. of Eq. (47). Another is the modification of the evolution of $\delta_{m}$ due to the change of the effective gravitational constant $G_{\text {eff }}$. The growth index $\gamma$ of matter perturbations is linked to the parameters $\Sigma$ and $\eta[13]$. Thus two parameters $(\Sigma, \eta)$ will be useful to detect the signature of modified gravity theories from the future survey of weak lensing.

It can happen that the scales of weak lensing are in the region of non-linear clustering, in which case we need to map the linear power spectrum of the lensing potential into a non-linear one. In the context of modified gravity theories mapping formulas have not been well known. We leave the analysis of such non-linear regimes in weak lensing for future work.

In Einstein gravity with the Lagrangian density $f=$ $R / 8 \pi G+2 p(\phi, X)$ we obtain the standard equation for matter perturbations:

$$
\delta_{m}^{\prime \prime}+\left(\frac{1}{2}-\frac{3}{2} w_{\mathrm{eff}}\right) \delta_{m}^{\prime}-\frac{3}{2} \Omega_{m} \delta_{m}=0,
$$

where a prime represents a derivative with respect to $N=\ln a$, and

$$
w_{\mathrm{eff}}=-1-\frac{2}{3} \frac{H^{\prime}}{H}, \quad \Omega_{m}=\frac{\rho_{m}}{3 F H^{2}} .
$$

If $w_{\text {eff }}$ and $\Omega_{m}$ are constants then the solution for Eq. (48) is

$$
\delta_{m}=c_{+} a^{n_{+}}+c_{-} a^{n-},
$$

where

$$
n_{ \pm}=\frac{1}{4}\left[3 w_{\mathrm{eff}}-1+\sqrt{\left(3 w_{\mathrm{eff}}-1\right)^{2}+24 \Omega_{m}}\right] .
$$

\footnotetext{
${ }^{1}$ In Ref. [13] the authors used a dimensionless function $\bar{F}=8 \pi G F$, where $G$ is a bare gravitational constant. Then one has $\Sigma=$ $G / \bar{F} G_{0}$.
}

One has $w_{\text {eff }} \simeq 0$ and $\Omega_{m} \simeq 1$ during a matter-dominated epoch provided that the contribution of the scalar field is negligible. Hence the matter perturbation grows as $\delta_{m} \propto$ $a \propto t^{2 / 3}$. However the evolution of $\delta_{m}$ is modified once the energy density of the scalar field becomes important relative to the matter density.

At the end of this section we consider the Brans-Dicke theory [29]:

$$
f(R, \phi, X)=\frac{\phi}{8 \pi} R+\frac{\omega_{\mathrm{BD}}}{4 \pi \phi} X
$$

where $\omega_{\mathrm{BD}}$ is a Brans-Dicke parameter. In this case the effective gravitational constant is given by

$$
G_{\mathrm{eff}}=\frac{1}{\phi} \frac{4+2 \omega_{\mathrm{BD}}}{3+2 \omega_{\mathrm{BD}}} .
$$

which agrees with the result in Ref. [30]. We have $G_{\text {eff }} \rightarrow$ $1 / \phi$ in the General Relativity (GR) limit $\left(\omega_{\mathrm{BD}} \rightarrow \infty\right)$. The deviation from GR is significant when $\omega_{\mathrm{BD}}$ is not much larger than unity. When $\omega_{\mathrm{BD}}=0$, for example, the effective gravitational constant is $4 / 3$ times larger than that in the GR case. This modifies the evolution of matter perturbations. However local gravity experiments place the bound on the present value of the Brans-Dicke parameter as $\omega_{\mathrm{BD}, 0}>4 \times 10^{4}$ [31]. This shows that unless $\omega_{\mathrm{BD}}$ is very much smaller than the present value during the matter epoch it is difficult to see the signature of modified gravity in the large-scale structure formation.

\section{IV. $f(R)$ GRAVITY}

In this section we study the evolution of matter perturbations in modified gravity theories where $f$ is the function of $R$ only. In this case the effective gravitational constant is given by

$$
G_{\mathrm{eff}}=\frac{1}{8 \pi F} \frac{1+4 \frac{k^{2}}{a^{2} R} m}{1+3 \frac{k^{2}}{a^{2} R} m},
$$

where

$$
m=\frac{R F_{, R}}{F} .
$$

The parameter $m$ was first introduced in Ref. [24]. This characterizes the deviation from the $\Lambda$ CDM model $(f(R)=R / 8 \pi G-\Lambda)$. The anisotropic parameter $\eta$ is given by

$$
\eta=\frac{2 \frac{k^{2}}{a^{2} R} m}{1+2 \frac{k^{2}}{a^{2} R} m} .
$$

In what follows we shall consider two different situations: (i) $\frac{k^{2}}{a^{2} R} m \gg 1$ and (ii) $\frac{k^{2}}{a^{2} R} m \ll 1$. 


\section{A. $\frac{k^{2}}{a^{2} R} m \gg 1$}

In this case one has $G_{\text {eff }} \simeq \frac{1}{8 \pi F} \frac{4}{3}$, which thus corresponds to Brans-Dicke theory with $\omega_{\mathrm{BD}}=0$ [33], see Eq. (53). Note that the anisotropic parameter is of order unity $(\eta \simeq 1)$ in such a case. Since the condition $k^{2} / a^{2} R \gg 1$ holds under the sub-horizon approximation $(k \gg a H)$, one can in fact realize $\frac{k^{2}}{a^{2} R} m \gg 1$ provided that $m$ is not very much smaller than unity. Then from Eq. (48) the matter perturbation equation is approximately given by

$$
\delta_{m}^{\prime \prime}+\left(\frac{1}{2}-\frac{3}{2} w_{\mathrm{eff}}\right) \delta_{m}^{\prime}-2 \Omega_{m} \delta_{m}=0,
$$

where $w_{\text {eff }}$ and $\Omega_{m}$ are defined in Eq. (49).

In order to estimate the evolution of $\delta_{m}$ and $\Phi$ analytically, let us consider the constant $m$ model. i.e.,

$$
f(R)=\alpha R^{1+m}-\Lambda,
$$

where $\alpha$ and $\Lambda$ are constants. In Ref. [24] it was shown that the matter-dominated epoch corresponds to a fixed point " $P_{5}$ " satisfying

$$
w_{\mathrm{eff}}=-\frac{m}{1+m}, \quad \Omega_{m}=1-\frac{m(7+10 m)}{2(1+m)^{2}},
$$

where $|m| \ll 1$.

Plugging Eq. (59) into Eq. (57), we obtain the solution for $\delta_{m}$ in the form (50) with

$$
n_{ \pm}=\frac{-(1+4 m) \pm \sqrt{(3+4 m)(11-28 m)}}{4(1+m)} .
$$

Since the growing mode corresponds to the power-law index $n_{+}$, the matter perturbation evolves as

$$
\delta_{m} \propto a^{n_{+}} \propto t^{\tilde{n}_{+}},
$$

where

$$
\tilde{n}_{+}=\frac{\sqrt{(3+4 m)(11-28 m)}-1-4 m}{6} .
$$

In Ref. [24] it was found that the viable matter epoch exists only for positive $m$ close to 0 . In the case of negative $m$ the matter point $P_{5}$ is unstable against perturbations around the fixed point. When $m=-1 / 4$ one has $w_{\text {eff }}=1 / 3$ and $\Omega_{m}=2$, which corresponds to a $\phi$ matter-dominated epoch ( $\phi \mathrm{MDE})$ [32]. For the models $f(R)=R-\beta / R^{n}(n>0)$ it was shown in Ref. [32] that the standard matter era is replaced by the $\phi \mathrm{MDE}$. From Eq. (60) we find that the matter perturbation evolves as $\delta_{m} \propto a^{2} \propto t$, which grows more rapidly than in the standard case $\left(\delta_{m} \propto a \propto t^{2 / 3}\right)$.

The power-law indices $n_{+}$and $\tilde{n}_{+}$are positive for $0<m<(\sqrt{73}-3) / 16$, whereas they are negative for $(\sqrt{73}-3) / 16<m<11 / 28$. When $m>11 / 28$ the matter perturbation exhibits a damped oscillation. Both $n_{+}$ and $\tilde{n}_{+}$get larger as $m$ decreases to zero. However we have to caution that we can not take the limit $m \rightarrow 0$ because of the breakdown of the condition $\frac{k^{2}}{a^{2} R} m \gg 1$. In this limit the evolution of $\delta_{m}$ is no longer described by the solution (61).

From Eq. (41) we find that the gravitational potential evolves as

$$
\Phi \propto t^{p_{+}}, \quad p_{+}=\frac{\sqrt{(3+4 m)(11-28 m)}+4 m-5}{6} .
$$

One has $p_{+}>0$ for $0<m<1 / 4$ and $p_{+}<0$ for $1 / 4<m<11 / 28$. Hence $\Phi$ is not constant except for the special case $m=1 / 4$ and the $\phi \mathrm{MDE}$ case $m=-1 / 4$. The variation of the gravitational potential leads to an Integrated-Sachs-Wolfe effect in the CMB spectrum. Thus it should be possible to constrain the magnitude of $m$ from CMB observations.

\section{B. $\frac{k^{2}}{a^{2} R} m \ll 1$}

Let us next consider the case in which the condition, $\frac{k^{2}}{a^{2} R} m \ll 1$, is satisfied on the scales around which large-scale structure is formed. In this case one has $G_{\text {eff }} \simeq \frac{1}{8 \pi F}\left(1+\frac{k^{2}}{a^{2} R} m\right)$ and $\eta \simeq 2 \frac{k^{2}}{a^{2} R} m$. The matter perturbation equation is approximately given by

$$
\delta_{m}^{\prime \prime}+\frac{1}{2} \delta_{m}^{\prime}-\frac{3}{2}\left(1+\frac{k^{2}}{a^{2} R} m\right) \delta_{m}=0 .
$$

Since the condition $m \ll \frac{k^{2}}{a^{2} R} m \ll 1$ holds under the subhorizon approximation, we only pick up the correction terms that contain $\frac{k^{2}}{a^{2} R} m$.

Expressing the solutions of this equation in the form $\delta_{m}=\exp \left(\int \omega \mathrm{d} N\right)$ and using the approximation $\left|\omega^{\prime}\right| \ll$ $\omega^{2}$, we obtain the growing-mode solution

$$
\omega_{+}=1+\frac{3}{5} \frac{k^{2}}{a^{2} R} m .
$$

If $m$ is constant, the second term on the r.h.s. of this equation is proportional to $a=e^{N}$ during the matter era. Then the evolution of the matter perturbation is given by

$$
\delta_{m} \propto a^{1+\frac{3}{5} \beta} \propto t^{\frac{2}{3}\left(1+\frac{3}{5} \beta\right)},
$$

where

$$
\beta \equiv \frac{k^{2}}{a^{2} R N} m=C k^{2} m \frac{a}{\ln a} .
$$

Here we have introduced a constant $C$ satisfying the relation $1 /\left(a^{2} R\right)=C e^{N}$. The gravitational potential evolves as

$$
\Phi \propto(1+\beta) t^{\frac{2}{5} \beta} .
$$

In the limit $\beta \rightarrow 0$ one obtains the standard result: $\delta_{m} \propto t^{2 / 3}$ and $\Phi=$ constant. When $\beta$ is positive, the 
growth rates of $\delta_{m}$ and $\Phi_{m}$ are larger than in the standard case. If $\beta$ grows to the order of unity, the results (66) and (68) are no longer valid.

In order to satisfy the local gravity constraint (LGC), we require that the condition $\frac{k^{2}}{a^{2} R} m \ll 1$ holds at the present epoch. The severest constraint may be obtained by laboratory experiments in which a strong modification of gravity is not observed on the scales up to $\lambda_{k} \sim a / k \sim 1 \mathrm{~mm}$. This gives the following constraint

$$
m(z=0) \ll\left(\lambda_{k} / H_{0}^{-1}\right)^{2} \sim 10^{-58},
$$

where we used $R \sim H_{0}^{2}$ and $H_{0}^{-1} \sim 10^{28} \mathrm{~cm}$. Note that this agrees with the result in Ref. [34] that was derived by using the effective mass of a scalar-field potential in the Einstein frame (see Refs. [35] for recent works of LGC in $f(R)$ gravity). The condition (69) can be weakened by taking into account the fact that the scalar curvature $R$ in the regime of a local structure such as an earth is much larger than the cosmological one. In solar system experiments the scale $\lambda_{k}$ corresponds to a value around $\lambda_{k}=1 \mathrm{au}$, which is much larger than in the case of laboratory experiments. In such cases the constraint on $m$ becomes much weaker than the one given in Eq. (69), although it is not easy to obtain the values of $m$ close to the order of unity [34].

The variable $m$ generally changes with time apart from the model $f=\alpha R^{1+m}-\Lambda$ discussed above. One can consider models in which $m$ satisfies $\frac{k^{2}}{a^{2} R} m \gg 1$ during the matter epoch and then enters the regime $\frac{k^{2}}{a^{2} R} m \ll 1$ in the dark energy era with the decrease of $m$. The anisotropic stress parameter $\eta$ decreases from 1 to $2 \frac{k^{2}}{a^{2} R} m$ together with the change of the quantity $\Sigma$ given in Eq. (46). It will be of interest to place observational constraints on such models by using the future data of weak lensing as well as the LSS data.

\section{SCALAR-TENSOR GRAVITY}

In this section we shall consider scalar-tensor gravity with a Lagrangian density

$$
f(R, \phi, X)=F(\phi) R+2 p(\phi, X) .
$$

Note that this includes most of scalar-field dark energy models such as quintessence [7], k-essence [8] and tachyons [36]. In this case the effective gravitational constant $G_{\text {eff }}$ and the anisotropic parameter $\eta$ are given by

$$
\begin{aligned}
G_{\mathrm{eff}} & =\frac{1}{8 \pi F} \frac{2 p_{, X}+4 F_{, \phi}^{2} / F}{2 p_{, X}+3 F_{, \phi}^{2} / F} \\
\eta & =\frac{F_{, \phi}^{2}}{p_{, X} F+F_{, \phi}^{2}} .
\end{aligned}
$$

In the case of quintessence with the Lagrangian density $p=X-V(\phi)$ the above results agree with those obtained by Boisseau et al. [27] and by Amendola et al. [13].
One can study the evolution of perturbations in the Jordan frame as we have done in $f(R)$ gravity models. Alternatively the dynamics of perturbations can be understood by making a conformal transformation to the Einstein frame. This is particularly useful when we relate scalar-tensor models with coupled DE scenarios [21] extensively studied by many authors (see [6] for references). We shall make a conformal transformation [37]

$$
\tilde{g}_{\mu \nu}=\Omega g_{\mu \nu}, \quad \Omega=\sqrt{F},
$$

where a tilde denotes quantities in the Einstein frame. Then the action in the Einstein frame is given by

$$
\tilde{S}=\int \mathrm{d}^{4} \tilde{x} \sqrt{-\tilde{g}}\left[\frac{1}{2 \kappa^{2}} \tilde{R}+\tilde{p}(\phi, \tilde{X})+\tilde{\mathcal{L}}_{m}(\phi)\right]
$$

where $\kappa^{2}=8 \pi G$ ( $G$ is a bare gravitational constant) and

$$
\tilde{p}(\phi, \tilde{X})=\frac{3}{2}\left(\frac{F, \phi}{F}\right)^{2} \tilde{X}+\frac{1}{F^{2}} p(\phi, F \tilde{X}) .
$$

In what follows we shall use the unit $\kappa^{2}=1$, but we restore the gravitational constant $G$ when it is needed.

We also have the following relations

$$
\tilde{a}=\sqrt{F} a, \quad \mathrm{~d} \tilde{t}=\sqrt{F} \mathrm{~d} t, \quad \tilde{\rho}_{m}=\rho_{m} / F^{2} .
$$

Then the continuity equation (8) is transformed as

$$
\frac{\mathrm{d}}{\mathrm{d} \tilde{t}} \tilde{\rho}_{m}+3 \tilde{H} \tilde{\rho}_{m}=Q(\phi) \tilde{\rho}_{m} \frac{\mathrm{d} \phi}{\mathrm{d} \tilde{t}},
$$

where

$$
Q(\phi)=-\frac{F_{, \phi}}{2 F} .
$$

Hence the non-relativistic matter is coupled to the field $\phi$ through the coupling $Q(\phi)$.

The perturbations in the Einstein frame are related to those in the Jordan frame via [26]

$$
\tilde{\delta}_{m}=\delta_{m}-\frac{2 \delta F}{F}, \quad \tilde{\Phi}=\Phi+\frac{2 \delta F}{F} .
$$

Under the sub-horizon approximation one can regard $\tilde{\delta}_{m} \simeq \delta_{m}$, whereas the $2 \delta F / F$ contribution can not be neglected for the gravitational potential. Using the relation $\tilde{p}_{, \tilde{X}}=\frac{3}{2}\left(\frac{F, \phi}{F}\right)^{2}+\frac{p, X}{F}$ together with the definition of $Q$ given in Eq. (78), we find that Eq. (39) is written in terms of the quantities in the Einstein frame:

$$
\begin{aligned}
& \frac{\mathrm{d}^{2}}{\mathrm{~d} \tilde{t}^{2}} \tilde{\delta}_{m}+\tilde{H}\left(2+Q \frac{1}{\tilde{H}} \frac{\mathrm{d} \phi}{\mathrm{d} \tilde{t}}\right) \frac{\mathrm{d}}{\mathrm{d} \tilde{t}} \tilde{\delta}_{m} \\
& -4 \pi G\left(1+\frac{2 Q^{2}}{\tilde{p}, \tilde{X}}\right) \tilde{\rho}_{m} \tilde{\delta}_{m}=0 .
\end{aligned}
$$

In this section we use a prime to represent a derivative with respect to the number of $e$-foldings $\tilde{N}=\int \tilde{H} \mathrm{~d} \tilde{t}$ 
in the Einstein frame. Then the above equation can be written as

$\tilde{\delta}_{m}^{\prime \prime}+\left(2+\frac{\tilde{H}^{\prime}}{\tilde{H}}+Q \phi^{\prime}\right) \tilde{\delta}_{m}^{\prime}-\frac{3}{2} \tilde{\Omega}_{m}\left(1+\frac{2 Q^{2}}{\tilde{p}_{, \tilde{X}}}\right) \tilde{\delta}_{m}=0$

where $\tilde{\Omega}_{m}=8 \pi G \tilde{\rho}_{m} / 3 \tilde{H}^{2}$. This fully agrees with the result in coupled dark energy scenarios derived by Amendola [20] without any reference to the Jordan frame (see also Ref. [38]).

Equation (80) shows that the effective gravitational constant in the Einstein frame is given by

$$
\tilde{G}_{\mathrm{eff}}=G\left(1+\frac{2 Q^{2}}{\tilde{p}_{, \tilde{X}}}\right)=G \frac{2 p_{, X}+4 F_{, \phi}^{2} / F}{2 p_{, X}+3 F_{, \phi}^{2} / F}
$$

From Eqs. (36) and (79) the gravitational potential in the Einstein frame is

$$
\tilde{\Phi}=\frac{2 p_{, X}+3 F_{, \phi}^{2} / F}{2 p_{, X}+4 F_{, \phi}^{2} / F} \Phi,
$$

which satisfies the relation

$$
\tilde{\Phi}=-4 \pi G \frac{\tilde{a}^{2}}{k^{2}} \tilde{\rho}_{m} \tilde{\delta}_{m}
$$

The effective gravitational potential acting on the matter is not $\tilde{\Phi}$ but $\tilde{\Phi}^{*}=\tilde{\Phi}+Q \delta \phi=\Phi[20]$, i.e., that in the Jordan frame. In fact, from Eqs. (82) and (84), we obtain

$$
\tilde{\Phi}^{*}=-4 \pi \tilde{G}_{\mathrm{eff}} \frac{\tilde{a}^{2}}{k^{2}} \tilde{\rho}_{m} \tilde{\delta}_{m}
$$

In order to see the effect of an interaction between the field $\phi$ and the matter analytically it is convenient to study the constant $Q$ case, i.e.,

$$
F(\phi)=e^{-2 Q \phi} .
$$

In the case of an ordinary field with an exponential potential $\left[\tilde{p}(\phi, \tilde{X})=\tilde{X}-c e^{-\lambda \phi}\right]$, it is known that there exists a $\phi \mathrm{MDE}$ scaling solution satisfying $\tilde{\Omega}_{\phi}=\tilde{w}_{\text {eff }}=2 Q^{2} / 3=$ constant [21]. More generally the existence of scaling solutions restricts the form of the Lagrangian density to be

$$
\tilde{p}(\phi, \tilde{X})=\tilde{X} g(Y), \quad Y=\tilde{X} e^{\lambda \phi}
$$

where $g$ is an arbitrary function and $\lambda$ is a constant quantity [39]. It was further shown in Ref. [40] that the $\phi \mathrm{MDE}$ exists for the models of the type

$$
g(Y)=c_{0}+\sum_{n>0} c_{n} Y^{-n}
$$

where $c_{0}(>0)$ and $c_{n}$ are constants. Note that quintessence with an exponential potential corresponds to the case $g(Y)=1-c_{1} / Y$. For the models (88) we have the following relations during the $\phi \mathrm{MDE}[40]$ :

$$
\tilde{\Omega}_{\phi}=\tilde{w}_{\mathrm{eff}}=\frac{2 Q^{2}}{3 c_{0}}, \quad \phi^{\prime}=-\frac{2 Q}{c_{0}}, \quad \tilde{p}_{, \tilde{X}}=c_{0},
$$

where $\tilde{\Omega}_{\phi}$ is an energy fraction of the scalar field satisfy$\operatorname{ing} \tilde{\Omega}_{\phi}+\tilde{\Omega}_{m}=1$.

Then from Eq. (81), the solution for matter perturbations during the $\phi \mathrm{MDE}$ is given by the form (50) with the power-law indices

$$
\tilde{n}_{+}=1+\frac{2 Q^{2}}{c_{0}}, \quad \tilde{n}_{-}=-\frac{3}{2}+\frac{Q^{2}}{c_{0}} .
$$

Since the scale factor grows as $\tilde{a} \propto \tilde{t}^{\frac{2 c_{0}}{3 c_{0}+2 Q^{2}}}$, the evolution of the matter perturbation in the Einstein frame is given by

$$
\tilde{\delta}_{m} \propto \tilde{a}^{1+\frac{2 Q^{2}}{c_{0}}} \propto \tilde{t}^{\frac{2 c_{0}+4 Q^{2}}{3 c_{0}+2 Q^{2}}}
$$

From Eq. (89) we obtain

$$
\phi=\phi_{0}-\frac{4 Q}{3 c_{0}+2 Q^{2}} \ln \tilde{t} .
$$

Using the relations (76) between two frames we find

$$
t \propto \tilde{t}^{\frac{3 c_{0}-2 Q^{2}}{3 c_{0}+2 Q^{2}}}, \quad a \propto \tilde{t}^{\frac{2 c_{0}-4 Q^{2}}{3 c_{0}+2 Q^{2}}} .
$$

Hence the evolution of matter perturbations in the Jordan frame is given by

$$
\delta_{m} \propto a^{\frac{2 c_{0}+4 Q^{2}}{2 c_{0}-4 Q^{2}}} \propto t^{\frac{2 c_{0}+4 Q^{2}}{3 c_{0}-2 Q^{2}}} .
$$

Thus, in the presence of the coupling $Q$, the growth rate of matter perturbations is larger than in the case of Einstein gravity. From Eqs. (84) and (85) we find that the gravitational potential is constant in both Jordan and Einstein frames:

$$
\Phi \propto t^{0}, \quad \tilde{\Phi} \propto \tilde{t}^{0}
$$

which is a rather peculiar property of the $\phi \mathrm{MDE}$. Recall that this property also holds for the $\phi \mathrm{MDE}$ solution in the $f(R)$ gravity.

From Eq. (75) the Lagrangian density in the Jordan frame corresponding to the $\phi \mathrm{MDE}$ solution is given by

$$
\begin{aligned}
f(R, \phi, X)= & e^{-2 Q \phi}\left[R+2\left(c_{0}-6 Q^{2}\right) X\right. \\
& \left.+\sum_{n>0} 2 c_{n} X^{1-n} e^{-n(\lambda+2 Q) \phi}\right] .
\end{aligned}
$$

Thus the field $\phi$ has a universal coupling $e^{-2 Q \phi}$. For the model $g(Y)=1-c_{1} / Y$ the above Lagrangian density can be viewed as the dilaton gravity with an exponential potential $V(\phi)=2 c_{1} e^{-(\lambda+2 Q) \phi}$. It is interesting that string 
theory can give rise to the $\phi \mathrm{MDE}$ solution along which $\Omega_{m}$ and $w_{\text {eff }}$ are constants in both Jordan and Einstein frames. We note that $Q$ is required to be smaller than the order of unity to reproduce a standard matter era [21], whereas string theory typically provides the coupling $Q$ of order one at the perturbative regime [41]. In the region $\phi \gg 1$, however, the coupling may become weak as in the context of a runaway dilaton scenario [42].

\section{CONCLUSIONS}

In this paper we derived the matter perturbation equation (39) with the effective gravitational constant (40) for a Lagrangian density $f(R, \phi, X)$ without a direct coupling between $R$ and $X$. This analysis covers most of modified gravity models proposed in the current literature and will be useful to detect the deviation from the $\Lambda$ CDM model from the future surveys such as weak lensing and LSS. We have also evaluated the anisotropic parameter $\eta$ and the quantity $\Sigma=q(1+\eta / 2)$ in order to confront the models with future observations of weak lensing, see Eqs. (43) and (46). We have applied our results to (i) $f(R)$ gravity and (ii) scalar-tensor gravity with the Lagrangian density $f(R)=F(\phi) R+2 p(\phi, X)$.

In $f(R)$ gravity models the effective gravitational constant has a scale-dependent term $\frac{k^{2}}{a^{2} R} m$, where $m=$ $R f_{, R R} / f_{, R}$ characterizes the deviation of the $\Lambda \mathrm{CDM}$ model. The local gravity constraint is satisfied for the models in which the condition, $\frac{k^{2}}{a^{2} R} m \ll 1$, holds for the scale of the order $a / k=1 \mathrm{~mm}$ at the present epoch. If we take the cosmological value $R \sim H_{0}^{2}$, this gives a very stringent constraint: $m(z=0) \ll 10^{-58}$. This is weakened by using a local value of $R$ much larger than $H_{0}^{2}$. One can consider models in which the condition, $\frac{k^{2}}{a^{2} R} m \gg 1$, holds during the matter epoch on the scales around which large-scale structure is formed. For the constant $m$ model $\left(f=\alpha R^{1+m}-\Lambda\right)$ we have analytically derived the evolution of matter perturbations $\delta_{m}$ as well as gravitational potentials $\Phi$ during the matter-dominated epoch. Even when $m \ll 1$ this is different from the evolution in Einstein gravity $\left(\delta_{m} \propto a\right.$ and $\Phi=$ constant), which will be useful to place constraints on the value $m$ from future high-precision observations.

The scalar-tensor gravity with the Lagrangian density $f(R)=F(\phi) R+2 p(\phi, X)$ correspond to coupled DE models in the Einstein frame with a coupling $Q(\phi)=$ $-F_{, \phi} / 2 F$ between the scalar field and dark matter. We reproduced the equation of matter perturbations in coupled DE scenarios with the k-essence Lagrangian density $\tilde{p}(\phi, \tilde{X})$ by making a conformal transformation to the Einstein frame. Since the evolution of perturbations in coupled DE models has been extensively studied in literature, it is convenient to pay attention to the relation between Jordan and Einstein frames in order to discuss perturbations in the scalar-tensor gravity. In fact, for the models in which the $\phi$ matter dominated epoch $(\phi \mathrm{MDE})$ is present, we analytically derived growth rates of perturbations in both Jordan and Einstein frames. We also obtained the form of the Lagrangian density in the Jordan frame giving rise to the $\phi \mathrm{MDE}$ solution.

The difference between $f(R)$ gravity and scalar-tensor gravity may be understood in the following way. Taking into account a mass $M$ of the perturbation in the field $\phi$, the effective gravitational constant in Eq. (82) is given by $[20]$

$$
\tilde{G}_{\mathrm{eff}} \simeq G\left(1+\frac{2 Q^{2}}{\tilde{p}_{, \tilde{X}}} e^{-M \ell}\right)
$$

where $\ell$ is a length scale. Here we assumed that the sound speed $c_{s}$ of the field $\phi$ is of order unity. In scalar-tensor models, even if the mass $M$ is very light as $M \sim H_{0}$, the second term on the r.h.s. of (97) can be much smaller than unity to satisfy the local gravity constraints by choosing a small coupling $Q^{2}=\left(F_{\phi} / 2 F\right)^{2} \ll 1$ (pro-

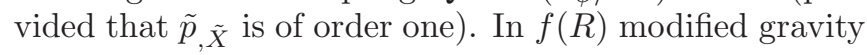
models, however, the coupling $Q$ is fixed as $Q=-1 / \sqrt{6}$ [32]. Hence we have to choose a heavy mass $M^{2} \simeq 1 / F_{, R}$ to satisfy the local gravity constraints, i.e., $e^{-M \ell} \ll 1$ for the scale $\ell \sim 1 \mathrm{~mm}$ [34]. In fact this is equivalent to choosing very small values of $m$ given in Eq. (69). In scalar-tensor gravity the coupling-dependent term is more important than the scale-dependent term provided that $M \ll k / a$, whereas in $f(R)$ gravity the scaledependent term plays a crucial role because of the fixed large coupling $Q$.

Although the perturbation equation we derived can be applied to many modified gravity models, it does not cover the DE models in which higher-order curvature corrections such as a Gauss-Bonnet (GB) term are present [43]. In Ref. [44] the equation of matter perturbations was derived in the presence of the GB term coupled to a scalar field $\phi$ to place constraints on GB DE models. A recent paper [45] shows that the GB energy fraction in the present universe is severely constrained by solar system tests. Moreover it is known that tensor perturbations typically show negative instabilities if the GB term is responsible for the accelerated expansion [46]. It will be of interest to extend our analysis to more general models that include such higher-order curvature corrections.

\section{ACKNOWLEDGMENTS}

This work is supported by JSPS (Grant No. 30318802). I thank Luca Amendola for useful discussions and drawing my attention to several important points. I am also grateful to Wayne Hu, Martin Kunz and David Polarski for useful comments. I also thank Istvan Laszlo for pointing out a typo in Eq. (42) that existed in the version 3 of this paper. 
[1] S. Perlmutter et al., Astrophys. J. 517, 565 (1999); A. G. Riess et al., Astron. J. 116, 1009 (1998); Astron. J. 117, 707 (1999).

[2] P. Astier et al., Astron. Astrophys. 447, 31 (2006); A. G. Riess et al., arXiv:astro-ph/0611572; W. M. WoodVasey et al., arXiv:astro-ph/0701041.

[3] D. N. Spergel et al. [WMAP Collaboration], Astrophys. J. Suppl. 148, 175 (2003); D. N. Spergel et al., arXiv:astro-ph/0603449.

[4] D. J. Eisenstein et al. [SDSS Collaboration], Astrophys. J. 633, 560 (2005).

[5] V. Sahni and A. A. Starobinsky, Int. J. Mod. Phys. D 9, 373 (2000); S. M. Carroll, Living Rev. Rel. 4, 1 (2001); V. Sahni, Lect. Notes Phys. 653, 141 (2004) [arXiv:astroph/0403324]; T. Padmanabhan, Phys. Rept. 380, 235 (2003); P. J. E. Peebles and B. Ratra, Rev. Mod. Phys. 75, 559 (2003); S. Nojiri and S. D. Odintsov, Int. J. Geom. Meth. Mod. Phys. 4, 115 (2007).

[6] E. J. Copeland, M. Sami and S. Tsujikawa, Int. J. Mod. Phys. D 15, 1753 (2006).

[7] Y. Fujii, Phys. Rev. D 26, 2580 (1982); C. Wetterich, Nucl. Phys B. 302, 668 (1988); B. Ratra and J. Peebles, Phys. Rev D 37, 321 (1988); T. Chiba, N. Sugiyama and T. Nakamura, Mon. Not. Roy. Astron. Soc. 289, L5 (1997); I. Zlatev, L. M. Wang and P. J. Steinhardt, Phys. Rev. Lett. 82, 896 (1999).

[8] T. Chiba, T. Okabe and M. Yamaguchi, Phys. Rev. D 62, 023511 (2000); C. Armendáriz-Picón, V. Mukhanov, and P. J. Steinhardt, Phys. Rev. Lett. 85, 4438 (2000).

[9] S. Capozziello, V. F. Cardone, S. Carloni and A. Troisi, Int. J. Mod. Phys. D 12, 1969 (2003); S. M. Carroll, V. Duvvuri, M. Trodden and M. S. Turner, Phys. Rev. D 70, 043528 (2004); S. Nojiri and S. D. Odintsov, Phys. Rev. D 68, 123512 (2003).

[10] J. P. Uzan, Phys. Rev. D 59, 123510 (1999); L. Amendola, Phys. Rev. D 60, 043501 (1999); T. Chiba, Phys. Rev. D 60, 083508 (1999); N. Bartolo and M. Pietroni, Phys. Rev. D 61, 023518 (2000); F. Perrotta, C. Baccigalupi and S. Matarrese, Phys. Rev. D 61, 023507 (2000); A. Riazuelo and J. P. Uzan, Phys. Rev. D 66, 023525 (2002); D. F. Torres, Phys. Rev. D 66, 043522 (2002); E. Elizalde, S. Nojiri and S. D. Odintsov, Phys. Rev. D 70, 043539 (2004); L. Perivolaropoulos, JCAP 0510, 001 (2005); D. A. Easson, JCAP 0702, 004 (2007); S. Nesseris and L. Perivolaropoulos, Phys. Rev. D 75, 023517 (2007).

[11] G. R. Dvali, G. Gabadadze and M. Porrati, Phys. Lett. B 485, 208 (2000); V. Sahni and Y. Shtanov, JCAP 0311, 014 (2003).

[12] P. Zhang, Phys. Rev. D 73, 123504 (2006); S. M. Carroll, I. Sawicki, A. Silvestri and M. Trodden, New J. Phys. 8, 323 (2006); R. Bean, D. Bernat, L. Pogosian, A. Silvestri and M. Trodden, Phys. Rev. D 75, 064020 (2007); Y. S. Song, W. Hu and I. Sawicki, Phys. Rev. D 75, 044004 (2007); B. Li and J. D. Barrow, Phys. Rev. D 75, 084010 (2007); T. Faulkner, M. Tegmark, E. F. Bunn and Y. Mao, arXiv:astro-ph/0612569; I. Sawicki and W. Hu, arXiv:astro-ph/0702278; K. Uddin, J. E. Lidsey and R. Tavakol, arXiv:0705.0232 [gr-qc]; S. Tsujikawa, arXiv:0709.1391 [astro-ph].

[13] L. Amendola, M. Kunz and D. Sapone, arXiv:0704.2421 [astro-ph].

[14] A. R. Cooray and D. Huterer, Astrophys. J. 513, 95 (1999); N. Sarbu, D. Rusin and C. P. Ma, Astrophys. J. 561, 147 (2001); K. H. Chae et al., Phys. Rev. Lett. 89, 151301 (2002); E. V. Linder, Phys. Rev. D 70, 043534 (2004); W. Hu and B. Jain, Phys. Rev. D 70, 043009 (2004); D. Jain, J. S. Alcaniz and A. Dev, Nucl. Phys. B 732, 379 (2006); M. Takada and B. Jain, Mon. Not. Roy. Astron. Soc. 348, 897 (2004); J. Albert et al. [SNAP Collaboration], arXiv:astro-ph/0507460; A. Refregier et al., arXiv:astro-ph/0610062.

[15] M. Tegmark et al. [SDSS Collaboration], Phys. Rev. D 69, 103501 (2004); K. Abazajian et al. [SDSS Collaboration], Astron. J. 128, 502 (2004); U. Seljak et al. [SDSS Collaboration], Phys. Rev. D 71, 103515 (2005); M. Tegmark et al., Phys. Rev. D 74, 123507 (2006).

[16] A. Shirata, T. Shiromizu, N. Yoshida and Y. Suto, Phys. Rev. D 71, 064030 (2005).

[17] O. Lahav, P. B. Lilje, J. R. Primack and M. J. Rees, Mon. Not. Roy. Astron. Soc. 251, 128 (1991).

[18] L. M. Wang and P. J. Steinhardt, Astrophys. J. 508, 483 (1998).

[19] E. V. Linder, Phys. Rev. D 72, 043529 (2005); D. Huterer and E. V. Linder, Phys. Rev. D 75, 023519 (2007); E. V. Linder and R. N. Cahn, arXiv:astro-ph/0701317.

[20] L. Amendola, Phys. Rev. Lett. 93, 181102 (2004).

[21] L. Amendola, Phys. Rev. D 62, 043511 (2000).

[22] G. Esposito-Farese and D. Polarski, Phys. Rev. D 63, 063504 (2001).

[23] R. Gannouji, D. Polarski, A. Ranquet and A. A. Starobinsky, JCAP 0609, 016 (2006).

[24] L. Amendola, R. Gannouji, D. Polarski and S. Tsujikawa, Phys. Rev. D 75, 083504 (2007).

[25] S. Tsujikawa, Phys. Rev. D 72, 083512 (2005).

[26] J. c. Hwang and H. Noh, Phys. Rev. D 71, 063536 (2005); Phys. Rev. D 66, 084009 (2002).

[27] B. Boisseau, G. Esposito-Farese, D. Polarski and A. A. Starobinsky, Phys. Rev. Lett. 85, 2236 (2000).

[28] C. Schimd, J. P. Uzan and A. Riazuelo, Phys. Rev. D 71, 083512 (2005).

[29] C. Brans and R. H. Dicke, Phys. Rev. 124, 925 (1961).

[30] T. Damour and K. Nordtvedt, Phys. Rev. D 48, 3436 (1993).

[31] B. Bertotti, L. Iess and P. Tortora, Nature 425, 374 (2003).

[32] L. Amendola, D. Polarski and S. Tsujikawa, Phys. Rev. Lett. 98, 131302 (2007).

[33] T. Chiba, Phys. Lett. B 575, 1 (2003).

[34] L. Amendola and S. Tsujikawa, arXiv:0705.0396 [astro$\mathrm{ph}$.

[35] G. J. Olmo, Phys. Rev. Lett. 95, 261102 (2005); J. A. R. Cembranos, Phys. Rev. D 73, 064029 (2006); A. L. Erickcek, T. L. Smith and M. Kamionkowski, Phys. Rev. D 74, 121501 (2006); V. Faraoni, Phys. Rev. D 74, 023529 (2006); A. F. Zakharov, A. A. Nucita, F. De Paolis and G. Ingrosso, Phys. Rev. D 74, 107101 (2006); I. Navarro and K. Van Acoleyen, JCAP 0702, 022 (2007); G. Allemandi and M. L. Ruggiero, arXiv:astro-ph/0610661; X. H. Jin, D. J. Liu and X. Z. Li, arXiv:astro-ph/0610854; T. Chiba, T. L. Smith and A. L. Erickcek, arXiv:astro-ph/0611867; 
P. J. Zhang, arXiv:astro-ph/0701662; W. Hu and I. Sawicki, arXiv:0705.1158 [astro-ph].

[36] A. Sen, JHEP 0204, 048 (2002); T. Padmanabhan, Phys. Rev. D 66, 021301 (2002); E. J. Copeland, M. R. Garousi, M. Sami and S. Tsujikawa, Phys. Rev. D 71, 043003 (2005).

[37] K. i. Maeda, Phys. Rev. D 39, 3159 (1989).

[38] L. Amendola, Phys. Rev. D 69, 103524 (2004).

[39] F. Piazza and S. Tsujikawa, JCAP 0407, 004 (2004); S. Tsujikawa and M. Sami, Phys. Lett. B 603, 113 (2004).

[40] L. Amendola, M. Quartin, S. Tsujikawa and I. Waga, Phys. Rev. D 74, 023525 (2006).

[41] M. Gasperini and G. Veneziano, Phys. Rept. 373, 1 (2003).

[42] M. Gasperini, F. Piazza and G. Veneziano, Phys. Rev. D 65, 023508 (2002); T. Damour, F. Piazza and G. Veneziano, Phys. Rev. Lett. 89, 081601 (2002).

[43] S. Nojiri, S. D. Odintsov and M. Sasaki, Phys. Rev. D 71, 123509 (2005); M. Sami, A. Toporensky, P. V. Tret- jakov and S. Tsujikawa, Phys. Lett. B 619, 193 (2005); G. Calcagni, S. Tsujikawa and M. Sami, Class. Quant. Grav. 22, 3977 (2005); S. Nojiri, S. D. Odintsov and M. Sami, Phys. Rev. D 74, 046004 (2006); S. Tsujikawa, Annalen Phys. 15, 302 (2006); B. M. N. Carter and I. P. Neupane, JCAP 0606, 004 (2006); T. Koivisto and D. F. Mota, Phys. Lett. B 644, 104 (2007); Phys. Rev. D 75, 023518 (2007); S. Tsujikawa and M. Sami, JCAP 0701, 006 (2007); B. M. Leith and I. P. Neupane, arXiv:hep-th/0702002.

[44] L. Amendola, C. Charmousis and S. C. Davis, JCAP 0612, 020 (2006).

[45] L. Amendola, C. Charmousis and S. C. Davis, arXiv:0704.0175 [astro-ph].

[46] A. De Felice, M. Hindmarsh and M. Trodden, JCAP 0608, 005 (2006); G. Calcagni, B. de Carlos and A. De Felice, Nucl. Phys. B 752, 404 (2006); Z. K. Guo, N. Ohta and S. Tsujikawa, Phys. Rev. D 75, 023520 (2007). 